\title{
A current perspective on stereotactic body radiation therapy for pancreatic cancer
}

\author{
This article was published in the following Dove Press journal: \\ OncoTargets and Therapy \\ 31 October 2016 \\ Number of times this article has been viewed
}

\author{
Julian C Hong \\ Brian G Czito \\ Christopher G Willett \\ Manisha Palta \\ Department of Radiation Oncology, \\ Duke University, Durham, NC, USA
}

Correspondence: Manisha Palta Department of Radiation Oncology, Duke University, Box 3085, Durham, NC 27705, USA

$\mathrm{Tel}+9196685640$

Fax +9196687345

Email manisha.palta@duke.edu

\begin{abstract}
Pancreatic cancer is a formidable malignancy with poor outcomes. The majority of patients are unable to undergo resection, which remains the only potentially curative treatment option. The management of locally advanced (unresectable) pancreatic cancer is controversial; however, treatment with either chemotherapy or chemoradiation is associated with high rates of local tumor progression and metastases development, resulting in low survival rates. An emerging local modality is stereotactic body radiation therapy (SBRT), which uses imageguided, conformal, high-dose radiation. SBRT has demonstrated promising local control rates and resultant quality of life with acceptable rates of toxicity. Over the past decade, increasing clinical experience and data have supported SBRT as a local treatment modality. Nevertheless, additional research is required to further evaluate the role of SBRT and improve upon the persistently poor outcomes associated with pancreatic cancer. This review discusses the existing clinical experience and technical implementation of SBRT for pancreatic cancer and highlights the directions for ongoing and future studies.
\end{abstract}

Keywords: pancreatic cancer, stereotactic body radiation therapy, SBRT, radiation therapy

\section{Introduction}

Pancreatic cancer is associated with a poor prognosis. It is estimated that 53,070 new diagnoses of pancreatic cancer and 41,780 deaths will occur in the United States in 2016, thereby making it the fourth leading cause of cancer deaths for both men and women. ${ }^{1}$ Given the poor outcomes, significant efforts have been undertaken to improve the treatment of pancreatic cancer. The only curative therapy is resection; however, only $15 \%-20 \%$ of patients present with resectable disease. Patients are often asymptomatic initially and symptoms develop only after local or distant progression. ${ }^{2}$ Moreover, even among the favorable patients who undergo surgical resection, the median overall survival is $\sim 20$ months despite the use of adjuvant chemotherapy or chemoradiation. ${ }^{3-5}$

Patients with unresectable, locally advanced pancreatic cancer have limited treatment options, and typically chemotherapy and/or radiotherapy are the primary treatment modalities. Treatment strategies such as chemotherapy followed by chemoradiation (in patients without disease progression on chemotherapy) result in a median overall survival of 15-16 months. ${ }^{6,7}$ In patients with unresectable pancreatic cancer, progressive local and distant disease leads to mortality. A Johns Hopkins rapid autopsy study showed that among 55 patients with stage III disease, $72 \%$ had metastatic disease and $28 \%$ had local-only disease. ${ }^{8}$ These patterns of progression suggest a need for improved local and systemic treatments. Moreover, patients with even limited metastatic disease burden in this study often died due to progressive local disease. 
Given the low rates of local control with chemotherapy or chemoradiation, the use of stereotactic body radiation therapy (SBRT; perhaps best described as hypofractionated image-guided radiotherapy), comprising treatment with five fractions of radiation or less, has become an area of active investigation. SBRT represents an alternative to conventionally fractionated chemoradiotherapy in the treatment of locally advanced pancreatic cancer. There are additional, although limited, data for SBRT as a complementary treatment for resectable patients in the neoadjuvant and adjuvant settings. We review the historical development, techniques, and clinical outcomes of SBRT for pancreatic cancer. In addition, practical considerations in implementing this modality and the areas of active investigation and future avenues for pancreatic SBRT are discussed.

\section{Clinical evolution of SBRT in the treatment of pancreatic cancer}

SBRT implements highly conformal and precise delivery of radiotherapy to achieve ablative doses to treatment volumes over the course of one to five fractions. This technique is well established in the treatment of central nervous system and pulmonary malignancies and has been increasingly adopted as a potential approach in treating abdominal cancers, including liver neoplasms such as hepatocellular carcinoma, cholangiocarcinoma, and liver metastases, as well as pancreatic cancer. ${ }^{9}$ To date, there have been several early phase, prospective studies characterizing the safety and efficacy of SBRT in the treatment of patients with pancreatic cancer, primarily in the locally advanced, unresectable setting (Table 1). In addition, numerous retrospective series have been published.

The use of SBRT in the treatment of patients with locally advanced pancreatic cancer was first reported in a Phase I dose escalation study from investigators at Stanford in 2004. Fifteen patients were treated with single-fraction SBRT with doses escalating from 15 Gy to 20 Gy to 25 Gy using the Accuray CyberKnife system (Accuray Inc., Sunnyvale, CA, USA). ${ }^{10}$ No dose-limiting toxicity was observed and there were no local recurrences with a median follow-up of 11 months. Despite high local control rates, all patients progressed distantly. These results prompted a Phase II study of 45 Gy intensity-modulated radiotherapy (IMRT) with concurrent 5-fluorouracil followed by an SBRT boost of $25 \mathrm{~Gy}$ in one fraction. ${ }^{11}$ This study also showed high rates of local control (94\% 1-year freedom from local recurrence with a median follow-up of 23 weeks) but with much higher rates of toxicity, including two patients who experienced grade 3 acute gastrointestinal (GI) toxicity (gastroparesis requiring parenteral support; one before SBRT and one after SBRT). Despite enhanced rates of local control, patients continued to develop distant metastases with corresponding poor survival rates (median overall survival of 33 weeks).

Given this pattern of distant recurrence, follow-up studies from this group delivered gemcitabine before and following SBRT, again with 25 Gy in one fraction. ${ }^{12}$ Sixteen patients with locally advanced pancreatic cancer were included in this study with a similar outcome profile: good local control (three patients with local progression at 14, 16, and 21 months after SBRT) but accompanied by a high rate of duodenal toxicity, with five ulcers, one duodenal stenosis, and one perforation (44\% grade $2+$ and $12.5 \%$ grade $3+$ ).

Given these toxicities, a follow-up Phase II study from Stanford of 20 patients with locally advanced pancreatic cancer was conducted with priority given to meeting duodenal constraints. ${ }^{13}$ This study also transitioned treatment to linear accelerator (linac)-based SBRT with IMRT. The protocol continued to use a 25 Gy single fraction

Table I Prospective studies of stereotactic body radiation therapy for pancreatic cancer

\begin{tabular}{|c|c|c|c|c|c|c|}
\hline Study & Regimen & Patients (n) & $\begin{array}{l}\text { I-year local } \\
\text { control (\%) }\end{array}$ & $\begin{array}{l}\text { Median OS } \\
\text { (months) }\end{array}$ & $\begin{array}{l}\text { Acute toxicity } \\
\text { grade } 3+(\%)\end{array}$ & $\begin{array}{l}\text { Late toxicity } \\
\text { grade } 2+(\%)\end{array}$ \\
\hline Koong et al ${ }^{10}$ & I5-25 Gy/l fx & I5 LA or LR & 100 & 11 & 0 & NR \\
\hline Koong et al" & 45 Gy IMRT $+5-\mathrm{FU} \rightarrow 25 \mathrm{~Gy} / \mathrm{fx}$ & 16 LA & 94 & 8.3 & 13 & NR \\
\hline Høyer et al ${ }^{16}$ & $15 \mathrm{~Gy} \times 3$ & 22 LA & 57 & 5.4 & 79 grade $2+$ & 94 \\
\hline Schellenberg et $\mathrm{al}^{12}$ & Gemcitabine $\rightarrow 25 \mathrm{~Gy} / \mathrm{l} \mathrm{fx} \rightarrow$ gemcitabine & 16 LA & 100 & 11.4 & 6 & 47 \\
\hline Polistina et $\mathrm{al}^{17}$ & $10 \mathrm{~Gy} \times 3$ & $23 \mathrm{LA}$ & 50 & 10.6 & 0 & 0 \\
\hline Schellenberg et $\mathrm{al}^{13}$ & Gemcitabine $\rightarrow 25 \mathrm{~Gy} / \mathrm{I} \mathrm{fx} \rightarrow$ gemcitabine & 20 LA & 94 & 11.8 & 5 & 20 \\
\hline Tozzi et al ${ }^{46}$ & Gemcitabine $\rightarrow 45 \mathrm{~Gy} / 6 \mathrm{fx}$ or $36 \mathrm{~Gy} / 6 \mathrm{fx}$ & 30 LA or LR & 77 & 11 & 0 & 0 \\
\hline Gurka et al ${ }^{18}$ & Gemcitabine $\rightarrow 25 \mathrm{~Gy} / 5 \mathrm{fx} \rightarrow$ gemcitabine & 10 LA & 40 & 12.2 & 0 & 0 \\
\hline Herman et al ${ }^{19}$ & Gemcitabine $\rightarrow 33 \mathrm{~Gy} / 5 \mathrm{fx}$ & 49 LA & 78 & 13.9 & 12 & 11 \\
\hline
\end{tabular}

Note: Arrows demonstrate sequence of the treatment regimen.

Abbreviations: 5-FU, 5-fluorouracil; fx, fraction; Gy, gray; IMRT, intensity modulated radiotherapy; LA, locally advanced; LR, locally recurrent; NR, not reported; OS, overall survival. 
SBRT regimen. At most, $5 \%$ of the duodenum was permitted to receive $\geq 22.5 \mathrm{~Gy}$, and half or less of the duodenum at the planning target volume (PTV) was permitted to receive $\geq 12.5$ Gy. PTV margins of $2-5 \mathrm{~mm}$ were used in this study. The local control was $94 \%$ at 1 -year with a single grade 4 toxicity (duodenal perforation [5\%]), and three patients developed duodenal ulcers (grade 2, 15\%). The authors note that the rate of late gastrointestinal toxicity was comparable to conventionally fractionated chemoradiotherapy and intraoperative electron beam radiotherapy. ${ }^{14,15}$

In parallel with these studies, fractionated SBRT has been investigated in European prospective studies. A multiinstitutional Phase II study in Denmark investigated linac-based SBRT using 45 Gy in three fractions in a cohort of 22 patients. Despite fractionation, the study found poor local control rates (57\% at 1 year) and little palliative benefit with high rates of acute GI toxicity (79\% grade $2+$ toxicity following SBRT). ${ }^{16}$ In this study, patients were treated using abdominal compression and more generous PTV margins ( $5 \mathrm{~mm}$ in the transverse and $1 \mathrm{~cm}$ in the craniocaudal direction) than described in the Stanford series. Additionally, peritumoral edema was included in the target. These larger treatment volumes may, in part, account for the higher rates of toxicity seen in this study compared to the Stanford series.

Polistina et al reported their prospective experience using CyberKnife treatments for 23 patients receiving $30 \mathrm{~Gy}$ in three fractions coupled with 6 weeks of induction and adjuvant gemcitabine. Volumes for this study were defined as per the Stanford SBRT protocol, and the study yielded modest rates of toxicity with no grade $2+$ acute or late toxicities with good rates of local response. ${ }^{17}$ Five patients were assessed as having resectable disease following SBRT. Three of these patients underwent resection, two patients had an R0 resection without complication, and one patient achieved a pathologic complete response (pCR). Of note, the 1-year local control in this study was $50 \%$, lower than other reports from studies incorporating SBRT.

Similarly, Georgetown University investigators performed a feasibility trial investigating the safety of full-dose gemcitabine with SBRT delivered with 25 Gy in five daily fractions during week 4 of cycle 1 using CyberKnife treatments. In their cohort of 10 evaluable patients, a 40\% 1-year local control without acute grade 3 toxicity was reported. ${ }^{18}$ Moreover, this study demonstrated the feasibility of SBRT employed with full-dose gemcitabine.

Most recently, a multiinstitutional, prospective Phase II study was undertaken at Johns Hopkins, Stanford, and Memorial Sloan-Kettering, using a multifraction technique in patients with locally advanced pancreatic cancer. ${ }^{19}$ The primary endpoint of this study was to define the grade 2 toxicity rates of a fractionated regimen and compare this to the single fraction results from Stanford. ${ }^{12}$ Forty-nine patients received 33 Gy in five fractions with up to three doses of gemcitabine weekly before SBRT, followed by additional gemcitabine until disease progression. Radiation treatment plans were centrally reviewed by a principal investigator. Additionally, fiducial marker placement, respiratory motion management, and adherence to predefined dose constraints were required. Study results were consistent with previously reported studies, with $79 \%$ local control at 1 year and a median overall survival of 13.9 months. A lower rate of toxicity was reported versus single fraction treatment, with one acute $(2 \%)$ and three late $(6 \%)$ grade $3+$ GI toxicities. Quality of life endpoints from this study are pending, but the investigators reported no decline in the quality of life following SBRT, with significant improvement in pain scores. ${ }^{20}$

Although prospective data demonstrate safety and acceptable clinical outcomes in the treatment of locally advanced and unresectable pancreatic cancer, there is significantly less experience for SBRT in the neoadjuvant and adjuvant settings. While surgery remains the only curative option in these patients, overall outcomes are poor. ${ }^{3}$ The Harvard group conducted a Phase I study of 15 patients with resectable pancreatic cancer using proton therapy with dose escalating hypofractionationated treatment with concurrent capecitabine in the preoperative setting, reaching a final dose level of 25 Gy in five fractions without encountering any dose-limiting toxicities. ${ }^{21}$ Of note, the treatment volumes in this study included the primary tumor and regional nodes (celiac, porta hepatis, superior mesenteric artery and vein, and paraaortic through the third portion of the duodenum). This led to a Phase II study of 35 patients using the 25 Gy in five fractions regimen neoadjuvantly, showing a $4.1 \%$ grade 3 acute toxicity. Among the total 50 patients (from the Phase I and II studies), 39 patients underwent resection, with 31 (84\%) patients achieving R0 resection without 30-day mortality or anastamotic leak. ${ }^{22}$ The median survival was 27 months with a median follow-up of 38 months, and $16 \%$ of those patients undergoing surgical resection had a locoregional recurrence.

Approximately $30 \%$ of patients present with borderline resectable pancreatic cancer. Although data are variable, a meta-analysis of Phase II studies showed that one-third of patients with borderline resectable or unresectable disease receiving neoadjuvant therapy were able to undergo surgical resection. ${ }^{23}$ Patients with borderline resectable disease are 
usually treated with neoadjuvant therapy either chemotherapy or chemoradiation. ${ }^{6}$ There are few retrospective analyses describing the experience of SBRT in this setting. ${ }^{24-26}$ Among these, Mellon et al described a series of 159 patients, including 110 patients with borderline resectable pancreatic cancer. Patients received a median dose of 30 Gy to the tumor and 40 Gy to the vessel-tumor interface in five fractions. For the patients with borderline resectable disease, $51 \%$ of the patients were able to undergo surgical resection, with $96 \%$ of them achieving margin-negative resection. ${ }^{25}$ Four patients (7\%) had a pCR. Similarly, a retrospective review of the Johns Hopkins experience included 19 out of 88 total patients with unresectable or borderline resectable disease who were able to undergo surgical resection. Of them, 84\% of the patients had margin-negative resection and $16 \%$ of the patients had $\mathrm{pCR} .^{26}$

In the postoperative setting, a single retrospective study from the University of Pittsburgh reported on the outcome of 24 patients receiving adjuvant SBRT. ${ }^{27}$ Among these patients, all had close or positive margins, with $96 \%$ of patients receiving single fraction SBRT with a median dose of $24 \mathrm{~Gy}$.
No patients experienced grade $3+$ acute or late toxicities, and freedom from local progression was $66 \%$ at 1 year.

\section{Technical considerations}

SBRT requires highly conformal and precise radiation delivery with significant imaging and technical requirements. Early experiences of SBRT in the treatment of pancreatic cancer patients from the Stanford experience used frameless stereotactic radiosurgery with the Accuray CyberKnife system. Since then, linac-based SBRT has been studied with similar outcomes (Figure 1).

\section{Simulation}

Prior to simulation, fiducial markers are often placed in the tumor or in close proximity to the tumor to assist in target identification at simulation and treatment. These are typically placed via endoscopic ultrasound just prior to simulation. At time of radiation simulation, an upper Vac-Lok (CIVCO Medical Solutions, Coralville, IA) on an indexed wingboard, alpha cradle, or body fix is created for immobilization. Assessment and management of respiratory motion is recommended.
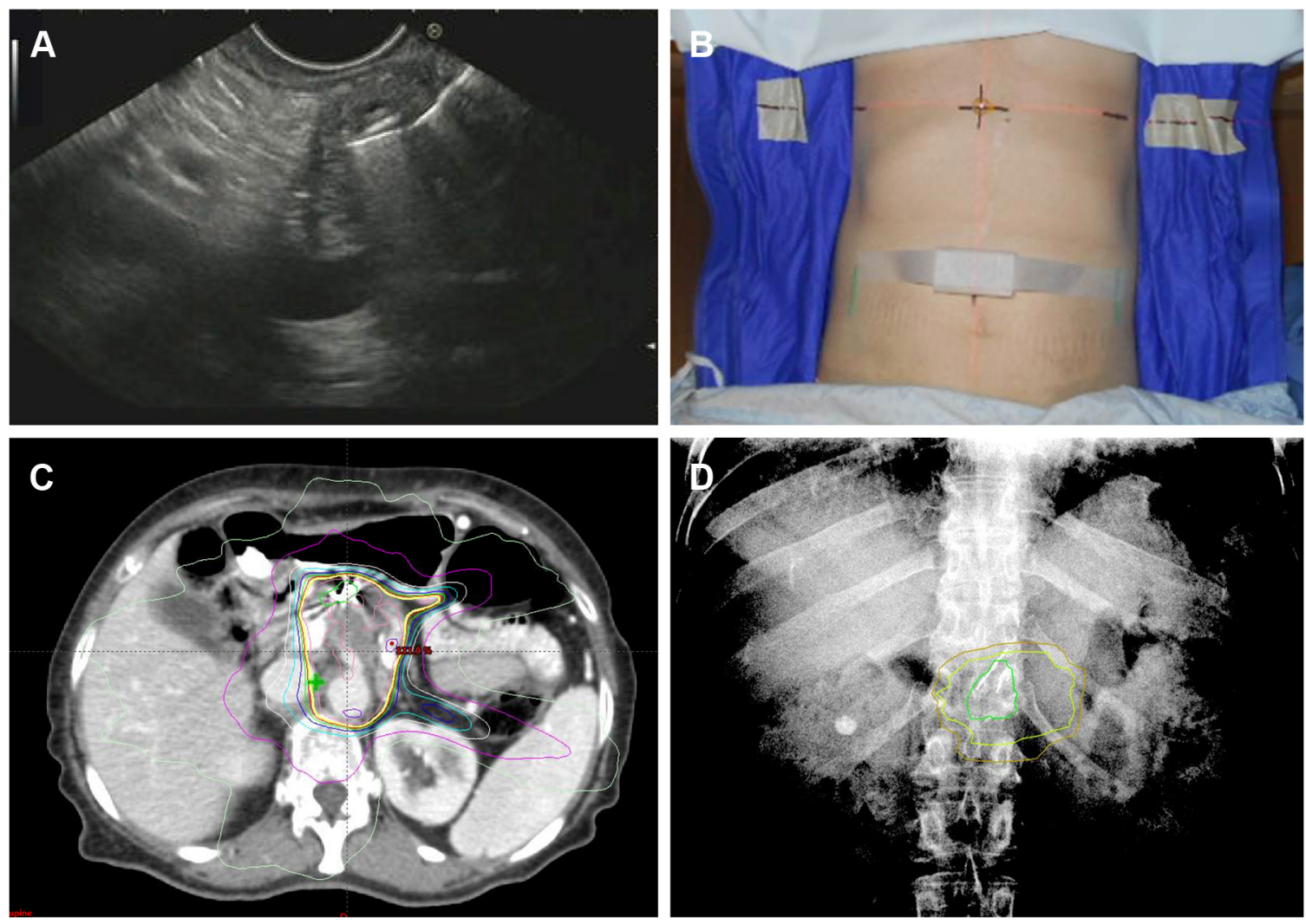

Figure I Workflow for pancreas stereotactic body radiation therapy.

Notes: (A) Fiducial marker placement by endoscopic ultrasound. (B) Simulation with immobilization and motion management (body fix and Varian Real-Time Position Management ${ }^{\mathrm{TM}}$; Palo Alto, CA, USA). (C) Treatment planning. (D) Image verification by kV fluoroscopy (pictured) or cone beam computed tomography. 
A four-dimensional computed tomography (CT) can be helpful to assess the magnitude of tumor motion with normal respiration. Motion management is challenging for abdominal tumors as there can be significant intrafraction and interfraction variations due to respiration that can be in the order of $2-3 \mathrm{~cm} .{ }^{28}$ If motion is $>3-5 \mathrm{~mm}$, respiratory motion management should generally be implemented with active breathing control, breath hold (ideally in end-expiration), or gating breath holding methods to minimize treatment volumes. There are data to suggest that end-expiration may allow for the most favorable anatomy to maximize therapeutic ratio. ${ }^{29}$ At our institution, we have not routinely used abdominal compression since this method has the potential to displace the duodenum and bowel toward the target volumes.

Patients are imaged using $\mathrm{CT} \pm$ magnetic resonance imaging (MRI) in supine position with arms up. Some institutions perform a positron emission tomography (PET)/CT simulation and utilize this for tumor response assessment post-SBRT. Prior retrospective analyses have found correlations between pre-SBRT PET parameters and clinical outcomes (discussed in "Future directions" section). ${ }^{30,31}$ The use of oral and IV contrast for better delineation of target and normal structures is recommended. Simulation imaging is typically performed with at least dual phasic contrast imaging with arterial and portal venous phases timed by bolus tracking software to appropriately time imaging with contrast administration. Use of multiphasic imaging allows greater delineation of the tumor, as these typically are seen best in contrast in the portal venous phase, with nonenhancement over the background of enhancing pancreatic tissues. ${ }^{32}$

\section{Definition of treatment volumes}

Gross tumor volume is identified based on simulation CT/MRI as well as other diagnostic imaging. With multiphasic imaging, the primary tumor and adjacent nodal disease is included in the gross target volume for each imaging sequence. These are combined to comprise an internal target volume (ITV), which should approximate the tumor motion identified on four-dimensional CT (if free breathing is used). The ITV is then expanded by $2-3 \mathrm{~mm}$ margin to the PTV. The PTV is then modified based on adjacent normal structures such as the duodenum, stomach and bowel and with additional coverage of regions away from the normal tissues (particularly in the retroperitoneum and along the vasculature). With a $2 \mathrm{~mm}$ margin between the PTV and normal organs, there may be selective underdosing of the PTV to respect normal tissue constraints. Additionally, an ITV contour for the fiducial markers or adjacent surgical clips may assist target alignment at the time of treatment.

\section{Treatment planning}

Ideally IMRT or volumetric modulated arc therapy should be used to deliver necessary doses to the target while sparing normal tissues. Use of flattening filter-free SBRT can decrease treatment time. ${ }^{33}$ GI structures, particularly the duodenum, are the main sources of potential toxicity. In the Stanford experience, patients undergoing 25 Gy $\times 1$ had a higher rate of duodenal toxicity correlating with V15, V20, and Dmax. ${ }^{13}$ A lower rate of toxicity was seen in the multiinstitutional, Phase II experience that used a dose of 33 Gy in five fractions. ${ }^{19}$ The normal tissue constraints described in this study are as follows: proximal duodenum, stomach, and small bowel with V15 Gy $<9$ cc, V20Gy $<3$ cc, and V33 Gy $<1$ cc; liver V12 Gy $<50 \%$; combined kidneys V12 $<75 \%$; and spinal cord V8 Gy $<1 \mathrm{cc}$.

\section{Treatment delivery}

Image-guided radiation therapy by $\mathrm{kV}$ or fluoroscopic on-board imaging and cone beam CT to verify the target and normal tissues prior to delivery of treatment are recommended. Fluoroscopy for alignment based on the fiducial markers is particularly important in target assessment as there are data to suggest that cone beam CT may underestimate the abdominal motion of pancreatic lesions. ${ }^{28}$ Cone beam CT should primarily be used to evaluate the location of normal tissues. Multiple breath holds may be required to acquire cone beam $\mathrm{CT}$ for patients where respiratory motion management is implemented. During treatment, we favor the use of prophylactic proton pump inhibitors and antiemetics.

\section{Future directions}

Selecting appropriate candidates for SBRT remains an area of active investigation. There are data to suggest that patterns of recurrence may be predicted based on radiographic, laboratory, or pathologic biomarkers. Given the promise of SBRT as a local therapy, these methods may allow better identification of patients who would benefit from local therapy. PET parameters have been of emerging interest in multiple disease sites, and metabolic tumor volume and total lesion glycolysis have been metrics of active investigation. Pre-SBRT SUVmax has previously been reported to be correlated with survival and progression-free survival. ${ }^{30}$ A subcohort of the previously mentioned multiinstitutional study had baseline PET imaging that was analyzed, finding a correlation between metabolic tumor volume and total lesion glycolysis with overall survival for patients undergoing SBRT. ${ }^{31}$ Similarly, laboratory biomarkers may have some utility with recent retrospective data 
to suggest correlation between pre-SBRT albumin levels and neutrophil-lymphocyte ratio with overall survival. ${ }^{34}$

Pathologic biomarkers are another active area of investigation. A rapid autopsy study from Johns Hopkins showed a correlation between the loss of SMAD4/DPC4 expression and greater propensity for metastatic disease. ${ }^{8}$ Within the same pathway, high transforming growth factor- $\beta 1$ plasma levels, the SMAD4 single-nucleotide polymorphism rs11354983, and high transforming growth factor- $\beta$ R2/SMAD4 expression are similarly correlated with poor outcomes. ${ }^{35}$ SMAD4 is a stratification factor, alongside CA19-9 levels, in ongoing cooperative group studies. ${ }^{36}$

Finally, there is a need for more effective adjunct therapies given the low overall survival and high rates of distant progression despite reasonable local control. Thus, incorporating surgical resection and systemic therapies to complement SBRT are active areas of investigation. In addition to prospective studies of SBRT as a neoadjuvant ${ }^{37}$ and adjuvant therapy, ${ }^{38}$ studies are actively combining traditional chemotherapy with neoadjuvant SBRT, such as FOLFIRINOX ${ }^{39,40}$ and gemcitabine/nab-paclitaxel. ${ }^{41}$ Beyond chemotherapy, there is also an increasing interest in the role of immune therapies, with ongoing studies of whole cell tumor vaccines. ${ }^{42-44}$ SBRT has spurred much interest as a potential complement in the form of immunosensitization in many disease sites and may further enhance these systemic therapies. ${ }^{45}$

\section{Conclusion}

There is a growing experience in the use of SBRT for the treatment of patients with pancreatic cancer. At present, there has been no prospective comparison of SBRT versus conventionally fractionated chemoradiation. The delivery of high-dose, highly conformal, and precise radiotherapy enables ablative therapy to unresectable pancreatic lesions, with data suggesting local control rates of at least $78 \%$. While continued advancements in imaging, target localization, and dosimetric parameters should improve toxicity profiles, further study with systemic therapies is required to improve the poor survival outcomes.

\section{Disclosure}

The authors report no conflicts of interest in this work.

\section{References}

1. Siegel RL, Miller KD, Jemal A. Cancer statistics, 2016. CA Cancer J Clin. 2016;66(1):7-30.

2. Vincent A, Herman J, Schulick R, Hruban RH, Goggins M. Pancreatic cancer. Lancet. 2011;378(9791):607-620.

3. Oettle H, Post S, Neuhaus P, et al. Adjuvant chemotherapy with gemcitabine vs observation in patients undergoing curative-intent resection of pancreatic cancer: a randomized controlled trial. JAMA. 2007;297(3):267-277.
4. Kalser MH, Ellenberg SS. Pancreatic cancer. adjuvant combined radiation and chemotherapy following curative resection. Arch Surg. 1985; 120(8):899-903.

5. Neoptolemos JP, Stocken DD, Bassi C, et al. Adjuvant chemotherapy with fluorouracil plus folinic acid vs gemcitabine following pancreatic cancer resection: a randomized controlled trial. JAMA. 2010; 304(10):1073-1081.

6. Huguet F, André T, Hammel P, et al. Impact of chemoradiotherapy after disease control with chemotherapy in locally advanced pancreatic adenocarcinoma in GERCOR phase II and III studies. J Clin Oncol. 2007; 25(3):326-331.

7. Hammel P, Huguet F, van Laethem J-L, et al. Effect of chemoradiotherapy vs chemotherapy on survival in patients with locally advanced pancreatic cancer controlled after 4 months of gemcitabine with or without erlotinib: the LAP07 randomized clinical trial. JAMA. 2016; 315(17):1844-1853.

8. Iacobuzio-Donahue CA, Fu B, Yachida S, et al. DPC4 gene status of the primary carcinoma correlates with patterns of failure in patients with pancreatic cancer. J Clin Oncol. 2009;27(11):1806-1813.

9. Lee MT, Kim JJ, Dinniwell R, et al. Phase I study of individualized stereotactic body radiotherapy of liver metastases. J Clin Oncol. 2009; 27(10):1585-1591.

10. Koong AC, Le QT, Ho A, et al. Phase I study of stereotactic radiosurgery in patients with locally advanced pancreatic cancer. Int J Radiat Oncol Biol Phys. 2004;58(4):1017-1021.

11. Koong AC, Christofferson E, Le Q-T, et al. Phase II study to assess the efficacy of conventionally fractionated radiotherapy followed by a stereotactic radiosurgery boost in patients with locally advanced pancreatic cancer. Int J Radiat Oncol Biol Phys. 2005;63(2):320-323.

12. Schellenberg D, Goodman KA, Lee F, et al. Gemcitabine chemotherapy and single-fraction stereotactic body radiotherapy for locally advanced pancreatic cancer. Int J Radiat Oncol Biol Phys. 2008; 72(3):678-686.

13. Schellenberg D, Kim J, Christman-Skieller C, et al. Single-fraction stereotactic body radiation therapy and sequential gemcitabine for the treatment of locally advanced pancreatic cancer. Int J Radiat Oncol Biol Phys. 2011;81(1):181-188.

14. de Lange SM, van Groeningen CJ, Meijer OWM, et al. Gemcitabineradiotherapy in patients with locally advanced pancreatic cancer. Eur $J$ Cancer. 2002;38(9):1212-1217.

15. Willett CG, Del Castillo CF, Shih HA, et al. Long-term results of intraoperative electron beam irradiation (IOERT) for patients with unresectable pancreatic cancer. Ann Surg. 2005;241(2):295-299.

16. Høyer M, Roed H, Sengelov L, et al. Phase-II study on stereotactic radiotherapy of locally advanced pancreatic carcinoma. Radiother Oncol. 2005;76(1):48-53.

17. Polistina F, Costantin G, Casamassima F, et al. Unresectable locally advanced pancreatic cancer: a multimodal treatment using neoadjuvant chemoradiotherapy (gemcitabine plus stereotactic radiosurgery) and subsequent surgical exploration. Ann Surg Oncol. 2010;17(8): 2092-2101.

18. Gurka MK, Collins SP, Slack R, et al. Stereotactic body radiation therapy with concurrent full-dose gemcitabine for locally advanced pancreatic cancer: a pilot trial demonstrating safety. Radiat Oncol. 2013; $8(1): 44$.

19. Herman JM, Chang DT, Goodman KA, et al. Phase 2 multi-institutional trial evaluating gemcitabine and stereotactic body radiotherapy for patients with locally advanced unresectable pancreatic adenocarcinoma. Cancer. 2015;121(7):1128-1137.

20. Moningi S, Marciscano AE, Rosati LM, et al. Stereotactic body radiation therapy in pancreatic cancer: the new frontier. Expert Rev Anticancer Ther. 2014;14(12):1461-1475.

21. Hong TS, Ryan DP, Blaszkowsky LS, et al. Phase I study of preoperative short-course chemoradiation with proton beam therapy and capecitabine for resectable pancreatic ductal adenocarcinoma of the head. Int J Radiat Oncol Biol Phys. 2011;79(1):151-157. 
22. Hong TS, Ryan DP, Borger DR, et al. A phase $1 / 2$ and biomarker study of preoperative short course chemoradiation with proton beam therapy and capecitabine followed by early surgery for resectable pancreatic ductal adenocarcinoma. Int J Radiat Oncol Biol Phys. 2014; 89(4):830-838.

23. Assifi MM, Lu X, Eibl G, Reber HA, Li G, Hines OJ. Neoadjuvant therapy in pancreatic adenocarcinoma: a meta-analysis of phase II trials. Surgery. 2011;150(3):466-473.

24. Rajagopalan MS, Heron DE, Wegner RE, et al. Pathologic response with neoadjuvant chemotherapy and stereotactic body radiotherapy for borderline resectable and locally-advanced pancreatic cancer. Radiat Oncol. 2013;8(1):254.

25. Mellon EA, Hoffe SE, Springett GM, et al. Long-term outcomes of induction chemotherapy and neoadjuvant stereotactic body radiotherapy for borderline resectable and locally advanced pancreatic adenocarcinoma. Acta Oncol. 2015;54(7):979-985.

26. Moningi S, Dholakia AS, Raman SP, et al. The role of stereotactic body radiation therapy for pancreatic cancer: a single-institution experience. Ann Surg Oncol. 2015;22(7):2352-2358.

27. Rwigema J-CM, Heron DE, Parikh SD, et al. Adjuvant stereotactic body radiotherapy for resected pancreatic adenocarcinoma with close or positive margins. J Gastrointest Cancer. 2012;43(1):70-76.

28. Jayachandran P, Minn AY, Van Dam J, Norton JA, Koong AC, Chang DT. Interfractional uncertainty in the treatment of pancreatic cancer with radiation. Int J Radiat Oncol Biol Phys. 2010;76(2):603-607.

29. Taniguchi CM, Murphy JD, Eclov N, et al. Dosimetric analysis of organs at risk during expiratory gating in stereotactic body radiation therapy for pancreatic cancer. Int J Radiat Oncol Biol Phys. 2013;85(4):1090-1095.

30. Schellenberg D, Quon A, Minn AY, et al. 18Fluorodeoxyglucose PET is prognostic of progression-free and overall survival in locally advanced pancreas cancer treated with stereotactic radiotherapy. Int J Radiat Oncol Biol Phys. 2010;77(5):1420-1425.

31. Dholakia AS, Chaudhry M, Leal JP, et al. Baseline metabolic tumor volume and total lesion glycolysis are associated with survival outcomes in patients with locally advanced pancreatic cancer receiving stereotactic body radiation therapy. Int J Radiat Oncol Biol Phys. 2014; 89(3):539-546.

32. Fletcher JG, Wiersema MJ, Farrell MA, et al. Pancreatic malignancy: value of arterial, pancreatic, and hepatic phase imaging with multidetector row CT. Radiology. 2003;229(1):81-90.

33. Scorsetti M, Alongi F, Castiglioni S, et al. Feasibility and early clinical assessment of flattening filter free (FFF) based stereotactic body radiotherapy (SBRT) treatments. Radiat Oncol. 2011;6(1):113.

34. Alagappan M, Pollom EL, von Eyben R, et al. Albumin and neutrophillymphocyte ratio (NLR) predict survival in patients with pancreatic adenocarcinoma treated with SBRT. Am J Clin Oncol. 2016. Epub 2016 Jan 11.
35. Javle M, Li Y, Tan D, et al. Biomarkers of TGF- $\beta$ signaling pathway and prognosis of pancreatic cancer. PLoS One. 2014;9(1):e85942.

36. Radiation Therapy Oncology Group. High or Standard Intensity Radiation Therapy after Gemcitabine Hydrochloride and Nab-paclitaxel in Treating Patients with Pancreatic Cancer That Cannot Be Removed by Surgery. Available from: https://clinicaltrials.gov/ct2/show/ NCT01921751. NCT01921751. Accessed May 23, 2016.

37. University of Rochester. Pilot Study of Short-Course Preoperative Stereotactic Body Radiation Therapy for Resectable Pancreatic Cancer. Available from: https:/clinicaltrials.gov/ct2/show/NCT02347618. NCT02347618. Accessed May 23, 2016.

38. University of Pittsburgh. Radiosurgery for Resected Pancreas. Available from: https://clinicaltrials.gov/ct2/show/NCT01357525. NCT01357525. Accessed May 23, 2016.

39. University of Maryland. Borderline Pancreas Study: FOLFIRINOX +SBRT (GCC 1324). Available from: https://clinicaltrials.gov/ ct2/show/NCT01992705. NCT01992705. Accessed May 23, 2016.

40. Emory University. Phase I Study of Stereotactic Body Radiation Therapy and FOLFIRINOX in the Neoadjuvant Therapy of Pancreatic Cancer. Available from: https://clinicaltrials.gov/ct2/show/ NCT01446458. NCT01446458. Accessed May 23, 2016.

41. Duke University. Gemcitabine/Nab-Paclitaxel with HIGRT in Resectable Pancreatic Cancer. Available from: https://clinicaltrials.gov/ct2/ show/NCT02318095. NCT02318095. Accessed May 23, 2016.

42. Lutz E, Yeo CJ, Lillemoe KD, et al. A lethally irradiated allogeneic granulocyte-macrophage colony stimulating factor-secreting tumor vaccine for pancreatic adenocarcinoma. A Phase II trial of safety, efficacy, and immune activation. Ann Surg. 2011;253(2):328-335.

43. Laheru D, Lutz E, Burke J, et al. Allogeneic granulocyte macrophage colony-stimulating factor-secreting tumor immunotherapy alone or in sequence with cyclophosphamide for metastatic pancreatic cancer: a pilot study of safety, feasibility, and immune activation. Clin Cancer Res. 2008;14(5):1455-1463.

44. NewLink Genetics Corporation. Immunotherapy and SBRT Study in Borderline Resectable Pancreatic Cancer. Available from: https:// clinicaltrials.gov/ct2/show/NCT02405585. NCT02405585. Accessed May 23, 2016.

45. Finkelstein SE, Timmerman R, McBride WH, et al. The confluence of stereotactic ablative radiotherapy and tumor immunology. Clin Dev Immunol. 2011;2011:439752.

46. Tozzi A, Comito T, Alongi F, et al. SBRT in unresectable advanced pancreatic cancer: preliminary results of a mono-institutional experience. Radiat Oncol. 2013;8(1):148.
OncoTargets and Therapy

\section{Publish your work in this journal}

OncoTargets and Therapy is an international, peer-reviewed, open access journal focusing on the pathological basis of all cancers, potential targets for therapy and treatment protocols employed to improve the management of cancer patients. The journal also focuses on the impact of management programs and new therapeutic agents and protocols on

\section{Dovepress}

patient perspectives such as quality of life, adherence and satisfaction. The manuscript management system is completely online and includes a very quick and fair peer-review system, which is all easy to use. Visit http://www.dovepress.com/testimonials.php to read real quotes from published authors. 